\title{
Synthesis of Generalized Vertical-Plane Weather Radar Imagery Along Aircraft Flight Paths
}

\author{
Pravas R. Mahapatra \\ Department of Aerospace Engineering \\ Indian Institute of Science \\ Bangalore - 560 012, India \\ Email: pravas@aero.iisc.ernet.in
}

\author{
Vishnu V. Makkapati \\ Honeywell Technology Solutions Lab \\ 151/1, Doraisanipalya, Bannerghatta Road \\ Bangalore - 560 076, India \\ Email: vishnu.makkapati@honeywell.com
}

\begin{abstract}
A method for synthesizing weather pictures in vertical planes along aircraft flight paths is presented. The weather data are derived from a number of Doppler radars covering different parts of the flight path. The flight path consists of straight segments with arbitrary offset and orientation with respect to individual radar locations. The intersection of radar scanning cones with the vertical plane segments are described as families of radial lines or hyperbolas depending on whether the flight path passes over a given radar or is offset from it. Radar data may be stretched or interpolated between radials to achieve continuity and smoothness of display. Schemes are incorporated for optimally using redundant data from multiple radars. Both scalar and vector weather data may be displayed. Such display is of great value for flight planning.
\end{abstract}

\section{INTRODUCTION}

Doppler weather radars are the primary source of detailed multi-parameter weather situational data for aviation [1],[2]. Reflectivity (depicting local rainfall activity), radial velocity (showing the radial component of wind distribution) and Doppler spectrum width (indicating the distribution of turbulence in the atmosphere) constitute the raw or primary data products of weather radar chains such as the WSR-88D network and the more dedicated radars such as the Terminal Doppler Weather radar (TDWR). A number of other secondary or 'higher level' weather data products such as vector wind fields, accumulated rainfall etc. are derived from these primary products. Doppler radars with polarimetric capability generate a larger set of primary and derived data products.

High resolution weather data are often generated and displayed in the form of plan-position indicator (PPI) displays. Each PPI may display a single radar-derived parameter or a combination of multiple parameters. The PPI corresponding to a single radar displays the data field within the maximum range of the radar. To display data over larger geographical areas typically traversed by aircraft, data from multiple radars are required be mosaiced [3],[4]. Aircraft flight paths can be represented as straight lines or curves (typically straightsegmented) on a PPI or a mosaiced PPI. Such a plot enables the visualization of the expected weather along flight paths planned to be flown by pilots.

Because flight operations cover a range of altitudes, information on the vertical variation of weather parameters is important for aviation. However, PPI displays are incapable of depicting the vertical dimension of weather fields. To display the vertical structure of weather, a common format employed is the range height indicator (RHI) which shows the radar data along each azimuthal direction as the beam sweeps through various elevation angles in a vertical plane.

The conventional RHI depiction essentially represents a vertical section of 3-D weather through the radar location. However, in practice an aircraft flight path may have any general orientation and offset with respect to a given radar. A long flight path would typically consist of multiple straight segments each of random heading and length. It is extremely useful to pilots, controllers and aviation meteorologists to be able to see displays of weather parameters along a vertical surface passing through the aircraft flight path. This paper outlines an accurate and efficient scheme for the generation of such flight path weather displays.

\section{The RAdiAl And OfFSET RANGE-Height INDICATORS}

In a dedicated RHI scanning mode, the radar antenna maintains its azimuth angle and varies its elevation angle. The weather echo parameter of interest is mapped onto a vertical plane to obtain a cross section of the weather phenomenon. However, such dedicated RHI scanning interrupts the normal azimuthal scanning process and is not practical in general purpose weather surveillance situations. A more common method of generating RHI in modern radars is to synthesize it from volume scan data obtained by sequential circular scans at varying elevation angles. The synthesized RHI scan data have to be converted to a vertical-plane 2-D format appropriate for presentation in polar coordinates with respect to the radar location.

\section{A. Radial RHI}

The centerline of the radar beam moves along a conical surface while scanning at a constant elevation angle $\phi_{i}$. The intersection of this surface with the vertical plane through the radar location yields a pair of radials separated in azimuth by $\pi$ and in elevation by $\pi-2 \times \phi_{i}$. These radials constitute the 'generators' for the RHI display.

Radar volume scans are generated along radial lines with discrete spacing in elevation. It is necessary to adopt a 'gap- 


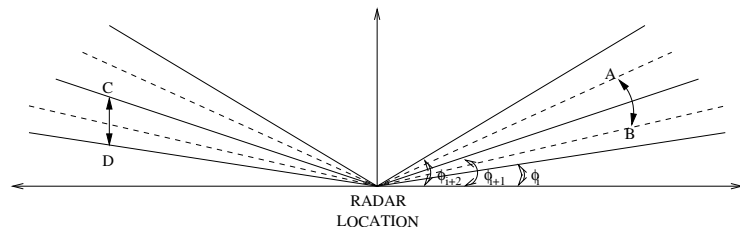

Fig. 1. Generators and interpolation scheme for radial RHI

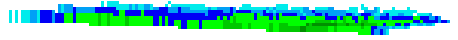

Fig. 2. Sample radial RHI synthesis

filling' or interpolation strategy to fill in the space between successive radials. The filling may be done by either stretching or interpolation. In Fig. 1 the solid radials represent the lines along which actual scan data are available, and the dotted radials represent the bisectors of the angle between successive data radials. In the stretching method, the data at any given range along a radial is stretched along a circular arc (e.g. $\mathrm{AB}$ in Fig. 1) extending between the bisectors on either side of the actual radial. In the interpolation method, the weather parameter value is interpolated between adjacent radials along the vertical line between them (e.g. CD in Fig. 1). Figure 2 shows a sample RHI along a vertical radial section through the radar.

\section{B. Offset RHI}

A schematic diagram showing intersection of the cone of radar sweep (at a given elevation angle) with an offset vertical plane through the flight path (called 'flight plane') is shown in Fig. 3. $\mathrm{O}$ is the center of the earth and $\mathrm{P}$ is the location of the radar on the earth's surface. The curve resulting from the intersection is in general a hyperbola (shown as a thick curve), which will degenerate to a pair of straight lines if the plane

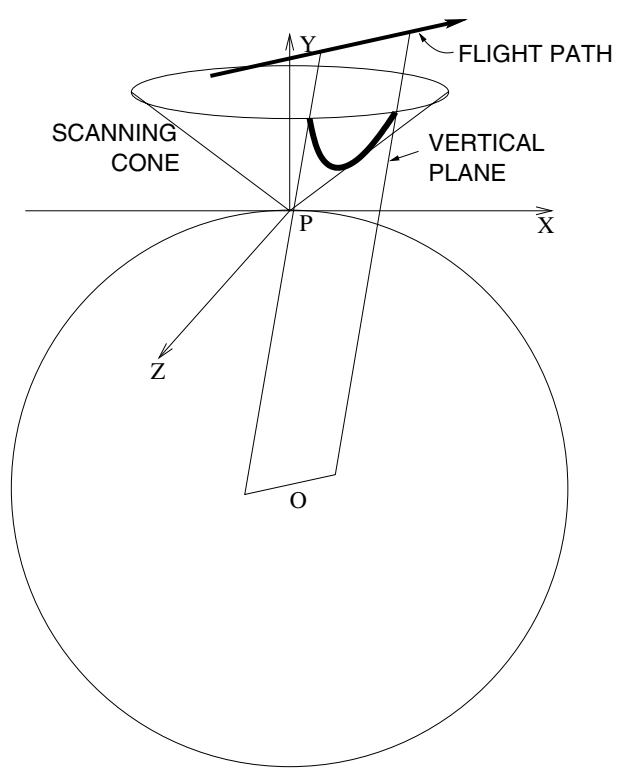

Fig. 3. Schematic for offset RHI

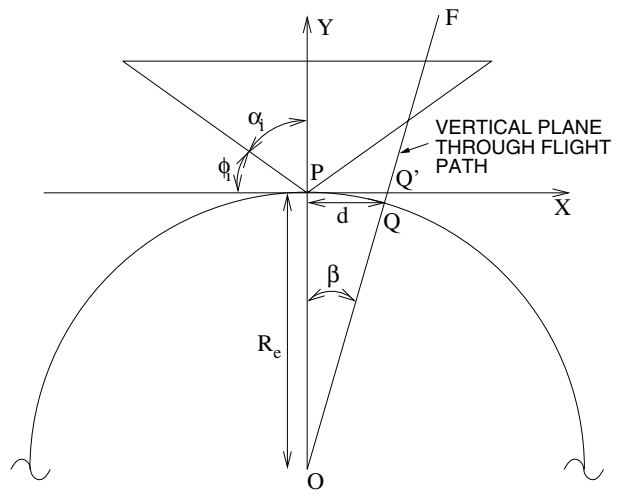

Fig. 4. Geometry for offset RHI

passes through the radar. A local coordinate system is set up wherein the $y$-axis is along the vertical at $\mathrm{P}$, and the $x$-axis is along the tangent to the earth at $\mathrm{P}$ in a direction such that the $x y$-plane is normal to the flight plane. The geometry showing the $x y$ planar section is depicted in Fig. 4. $\phi_{i}$ is the $i^{\text {th }}$ elevation angle of a volume scan, and $\alpha_{i}=\pi / 2-\phi_{i} . R_{e}$ is the radius of the earth. FQ is a side-view of the flight plane. By definition FQ, when extended, would pass through O. Although YP and FQ are both vertical, they would not be parallel because of their spatial separation. $\beta$ is the angle between OY and OF. The $x$-axis distance between the radar and the flight plane, $\mathrm{PQ}^{\prime}$, is $d=R_{e} \tan (\beta)$.

The scanning cone, with the radar as the origin, may be described by the equation $x^{2}+z^{2}=y^{2} \tan ^{2}\left(\alpha_{i}\right)$, and the flight plane is described as $x=y \times \tan (\beta)+d$. Solving these two equations, the equation for their intersection is obtained as:

$$
\begin{gathered}
\frac{\left(y-\frac{d \times \tan (\beta)}{\tan ^{2}\left(\alpha_{i}\right)-\tan ^{2}(\beta)}\right)^{2}}{\frac{d^{2}}{\tan ^{2}\left(\alpha_{i}\right)-\tan ^{2}(\beta)}+\frac{d^{2} \tan ^{2}(\beta)}{\left(\tan ^{2}\left(\alpha_{i}\right)-\tan ^{2}(\beta)\right)^{2}}} \\
-\frac{z^{2}}{d^{2}+\frac{d^{2} \tan ^{2}(\beta)}{\tan ^{2}\left(\alpha_{i}\right)-\tan ^{2}(\beta)}}=1
\end{gathered}
$$

Equation ( 1) represents a family of hyperbolas with $\alpha_{i}$ as parameter. However, equation ( 1) is still referenced to the radar location. To display the flight plane data, the hyperbolas must be transferred to a 2-D coordinate system lying on the flight plane itself. This is done through a series of transformations as follows: (1) shifting the origin of equation (1) to the center of the earth, (2) rotating the coordinates to align them with a latitude-longitude system, and (3) shifting the origin to the flight path projection point Q.

Each circular scan of the radar at an elevation $\phi_{i}$ contributes a hyperbola to the family, shown as solid lines in Fig. 5. These hyperbolas constitute the 'generators' for the RHI display. The radar azimuth and range corresponding to incremented points on a given parabola are calculated with reference to $\mathrm{P}$ and the corresponding values read from the scan line buffer after accounting for the inclination of the flight plane projection with the radar reference direction. 


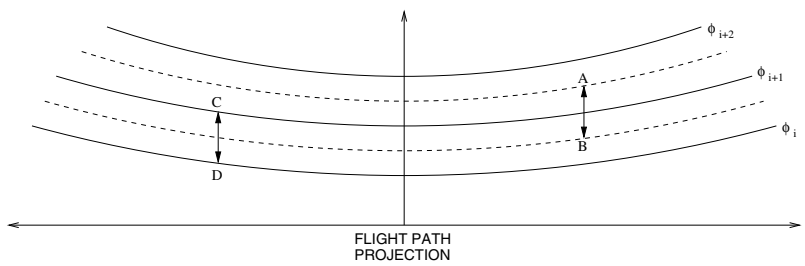

Fig. 5. Generators and interpolation scheme for offset RHI

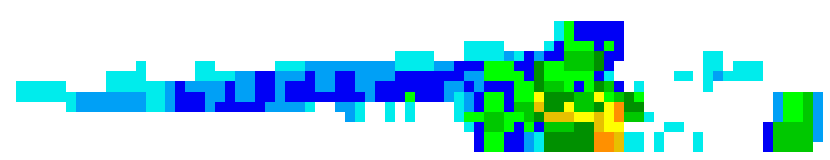

Fig. 6. Sample offset RHI synthesis

The values of range and azimuth may be used to read the necessary data from the scan line buffer and processed for display. As in the case of the radial RHI, the display data have to be interpolated between the parabolas corresponding to the scan elevations. Here again we obtain the intermediate parabolas corresponding to the bisectors between the successive scan elevations (dotted lines in Fig. 5), and use either a stretch or interpolation technique. However, both stretching and interpolation are carried out along a vertical line for reasons of tractability. Figure 6 shows a sample RHI along a vertical section offset from the radar.

\section{Multi-RadAR ARbitrary Plane RHI}

The generation of flight plane weather imagery gets considerably more complex when an aircraft plans to fly along a long path which typically consists of multiple straight line segments. In fact, in such cases the 'flight plane' actually consists of multiple sub-planes inclined to each other. Further, long flight paths generally would not fall within the field of view of a single radar, and data would have to be combined from multiple spatially separated radars. Some of the additional issues involved are discussed in the subsections below.

\section{A. Choice of Coordinate System}

Although it is common to use radar-centric coordinates to generate RHI data from a single radar, these are not adequate in multi-radar situations. We adopt the global latitudelongitude ('Lat-Long') grid frame to specify the locations of the radars themselves and also the aircraft flight paths in a universal way. If $\left(\lambda_{k}, \phi_{k}\right)$ and $\left(\lambda_{k+1}, \phi_{k+1}\right)$ denote the respective latitude and longitude of the $k^{\text {th }}$ and $(k+1)^{\text {th }}$ way points along the flight path, the angle $\Theta$ subtended by the $k^{\text {th }}$ straight line segment (between these way points) at the center of the earth is given as:

$$
\begin{array}{r}
\Theta=\cos ^{-1}\left(\cos \left(\lambda_{k}\right) \cos \left(\lambda_{k+1}\right) \cos \left(\phi_{k}-\phi_{k+1}\right)\right. \\
\left.+\sin \left(\lambda_{k}\right) \sin \left(\lambda_{k+1}\right)\right)
\end{array}
$$

The length of the $k^{\text {th }}$ flight segment equals $\left(R_{e}+h\right) \times \Theta$ where $h$ is the cruising altitude of the aircraft.

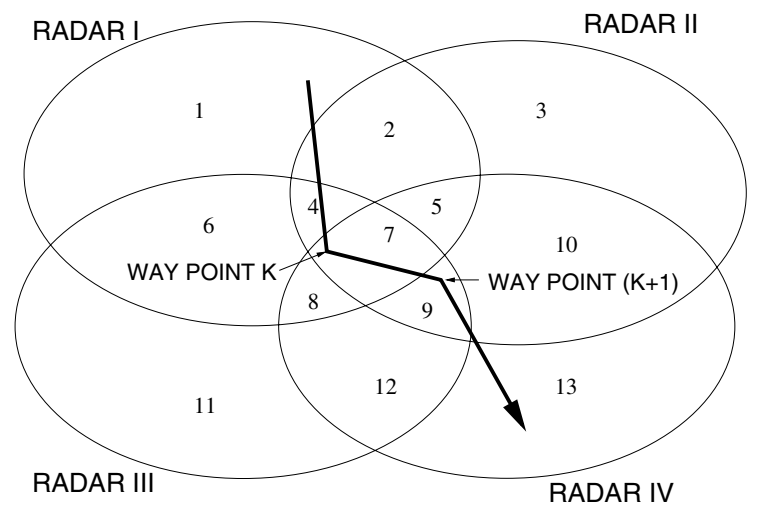

Fig. 7. Schematic of a 4-radar coverage region. The thick line represents a flight path.

\section{B. Determination of Projection Mode}

The location of all the weather radars likely to cover the flight path are assumed known in terms of their Lat-Long coordinates. A simple check is performed to determine if the flight segment passes over any of the radar locations. This is done by calculating the offset distance $d$ (Fig. 4) and applying a small threshold. For small offset distances (e.g. $5 \mathrm{~km}$ ) the radial RHI mode may be selected. Otherwise an offset RHI algorithm may be employed.

\section{Determination of Radar Coverage}

While flying through the coverage zones of multiple radars it is necessary to determine which of the radars would cover the flight path and over what segments. It is also necessary to determine how many and which radars are covering the flight path at a given instant. These issues are visualized with the help of Fig. 7 showing flight through a 4-radar domain. Table I lists the combination of radars that provide overlapping coverage of different regions.

A flight path through these radar coverage regions is shown as a curve consisting of straight legs, each of which is a segment of the great circle between two way points $k$ and $k+1$ with latitude-longitude $\left(\lambda_{k}, \phi_{k}\right)$ and $\left(\lambda_{k+1}, \phi_{k+1}\right)$ respectively. We have devised an algorithm to dynamically determine the identity of the radars from which data would be available for processing at any given instant. We set up an earth-centered polar coordinate system on the flight plane. The vertical through the projection of the radar location on the flight plane is the principal axis of the family of hyperbolas on that plane. Coverage by individual radars is ascertained by applying range criterion together with the finiteness of the flight plane.

\section{Scalar Data}

To display scalar data such as reflectivity at a given point, the average of the parameter values obtained from the overlapping radars is calculated and is displayed at that point. Alternatively, the maximum of the individual radar values may also be used for display. 
TABLE I

Multiple Radar Coverage Patterns

\begin{tabular}{|c|l|}
\hline Region & Overlapping radars \\
\hline 1 & I \\
\hline 2 & I and II \\
\hline 3 & II \\
\hline 4 & I, II, and III \\
\hline 5 & I, II, and IV \\
\hline 6 & I and III \\
\hline 7 & I, II, III, and IV \\
\hline 8 & I, III, and IV \\
\hline 9 & II, III, and IV \\
\hline 10 & II and IV \\
\hline 11 & III \\
\hline 12 & III and IV \\
\hline 13 & IV \\
\hline
\end{tabular}

\section{E. Vector Data}

The synthesis of vector data (e.g. wind velocities) from multiple radar observations is more complex. If a given point receives Doppler coverage from only one radar then only one component (towards the radar) of the wind vector will be available and hence the vector cannot be reconstructed. If two radars observe a given point, then two radial projections can be used to construct a planar vector (e.g. one lying in a horizontal plane), but its vertical component cannot be accurately estimated locally. However, a fair distribution of vertical velocities may be obtained by integrating the continuity equation from ground upwards [5],[6]. When Doppler observations at a point are available from three Doppler radars, the full 3-D vector can be synthesized from these projections and its vertical component displayed in the RHI. In the favorable situation where Doppler data are available from 4 or more radars, the 3 $D$ vector can be better estimated using a least-square approach, and its vertical component displayed. If $A X=B$ is a system of simultaneous linear (overdetermined) equations, then the least-squares solution is given by:

$$
A^{T} A X=A^{T} B
$$

where $A^{T}$ denotes the transpose of $\mathrm{A}$.

A convenient way for displaying vector data is by means of arrows depicting its magnitude and direction. This is clearly not possible for every pixel. We utilize each arrow to represent the average parameter over a matrix of pixels (or data grid points). In cases where the radar coverage pattern varies within the matrix itself, we determine the region (as given in Table I) that occupies the largest part of the matrix, and construct the vector from the components observed by the corresponding radars.

\section{SUMMARY}

A powerful and versatile method of generating weather imagery over the vertical section along flight paths has been presented in this paper. The following issues have been addressed:

- Long aircraft flight paths consisting of straight line segments spanning multiple radar coverage zones
- Arbitrary orientation and offset of each flight segment with respect to the radar(s) covering the segment

- Generation and representation of scalar as well as vector data products

- Variability of the number of radars covering a given location.

- Variability of the size and orientation of the radar resolution volumes of multiple radars at a given location.

- The conical nature of the radar scanning surfaces, and the changing geometry of their intersection with the flight plane at different offset distances from the radar location.

- Non-contiguous stacking of the scanning radar beams, i.e. presence of significant gaps between the scanning elevations of a radar.

- The curvature of the earth and its effects on data projection onto the flight plane

These issues have been addressed by incorporating a variety of innovative strategies/features such as

1) Specification of the aircraft flight path and radar locations in global latitude-longitude coordinate system

2) The use of the hyperbolic nature of the intersection of the conical radar scan surfaces with the flight plane

3) Correction for the inclination of the vertical flight plane with respect to the vertical through the radar, caused by the curvature of the earth

4) Adoption of an earth-centered polar coordinate system along the local flight plane

5) Use of rectangular area elements to compute and display velocity vectors

6) Automated enumeration of the number of radars observing a given point, and adaptive choice of the appropriate algorithm for combining data from these radars (including an algorithm for handling the variation of the number of observing radars within an area element)

7) Display of vector data as a field of arrows to provide a graphic feel of the vertical wind field

\section{REFERENCES}

[1] R. J. Doviak and D. S. Zrnic, Doppler Radar and Weather Observations, 2nd ed. San Diego, CA: Academic Press, 1993.

[2] P. R. Mahapatra, Aviation Weather Surveillance Systems: Advanced Radar and Surface Sensors for Flight Safety and Air Traffic Management. London, UK: IEE Press, 1999.

[3] P. Mahapatra and V. Makkapati, "Vector reconstruction and mosaicing from multiple doppler weather radar velocity data," in Proc. International Geoscience and Remote Sensing Symposium, vol. 6, Anchorage, Alaska, September 2004, pp. 3868 - 3871.

[4] J. Zhang, J. J. Gourley, K. Howard, and B. Maddox, "Three-dimensional gridding and mosaic of reflectivities from multiple wsr-88d radars," in Proc. 30th International Conference on Radar Meteorology, Munich, Germany, July 2001, pp. $719-721$.

[5] R. Watson and D. Bebbington, "Combining ground based meteorological radar data from multiple overlapping sites," in Proc. International Geoscience and Remote Sensing Symposium, vol. 3, Florence, Italy, July 1995 , pp. 1660 - 1662.

[6] T. Given and P. Ray, "Response of two dimensional dual Doppler radar wind synthesis," J. Atmos. Oceanic Tech., vol. 11, pp. 239 - 255, April 1994. 